\title{
An Illustrative Approach to Use SQL Functions: A Review
}

\author{
Kamalinder Kaur \\ Assistant Professor \\ Chandigarh Engineering College \\ Punjab, India
}

\begin{abstract}
This paper describes the function used in databases for performing the calculations, modifies the data items and does manipulations on data. It defines the functions, their syntax's and errors occurred during the process. Functions are applied on oracle having SQL and are illustrated through query processing.
\end{abstract}

Keywords- SQL; NVL; NULLIF; CASE; NVL2; DECODE.

\section{INTRODUCTION}

A SQL functions are brought up into oracle databases and are obtainable for its utilization in SQL queries.

\section{BENEFITS OF SQL FUNCTIONS}

The feature of SQL is its SQL Functions. These functions perform below tasks:
1) Executing calculations on data
2) Modification of individual data elements
3) Manipulate the results for collection of rows
4) Changing date and numbers to display
5) Conversion of data types of column

\section{TYPES OF SQL FUNCTIONS}

Single Row Function: These functions are applied on individual rows and then gives output on single row basis. The kinds of single-row functions are:

- $\quad$ On Character: Accepts character input and gives back both character and number digits.

- On Number: Accepts character input and gives back both character and numerical values.

- On Date: It works on values of the DATE data type. Almost all date functions outputs a value of DATE data type butthe MONTHS_BETWEEN gives a number.

- Conversion :altersvalue from one data type to another

- COALESCE, NVL, NULlif, CASE, NVL2, DECODE are common functions.

\section{Character Functions}

Character cases handling functions: (Lower, Initcapand Upper)

TABLE 1: Character Functions

\begin{tabular}{|l|l|}
\hline Function & Result \\
\hline LOWER('Hello Word') & hello word \\
\hline UPPER('Hello Word') & HELLO WORD \\
\hline INITCAP('Hello Word') & Hello Word \\
\hline
\end{tabular}

\section{EXECUTION OF QUERIES}

selectename"Emp_Name",lower(ENAME)"Lower_case",u pper(ename) "Upper_case", initcap(ename) "Initcap_case" from emp1 where empno in ('1','2','3','4').

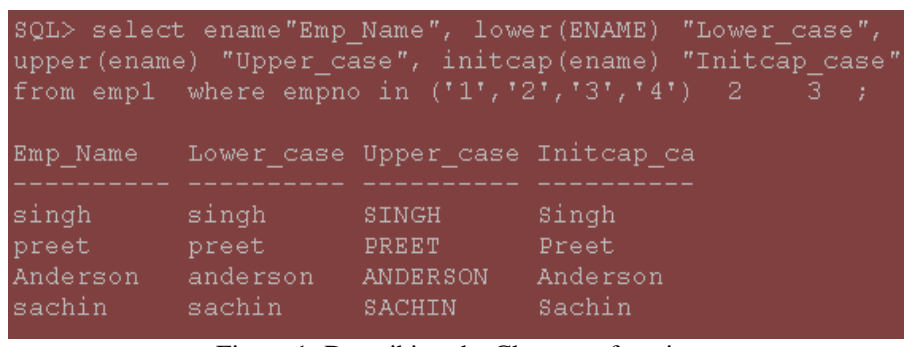

Figure 1: Describing the Character functions

This can work along with where clause: Select ename"Emp_Name",upper(job) "Job", hiredate from emp1 where ename='Anderson';

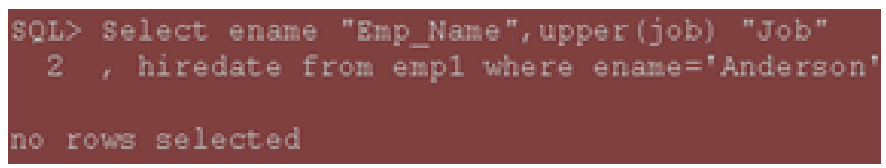

Figure 2: Where clause

Query with initcap,lower,upper function with where clause: 
select ename "Emp_Name", upper(job) "Job", hiredate from emp1 where initcap (ename) ='Anderson';

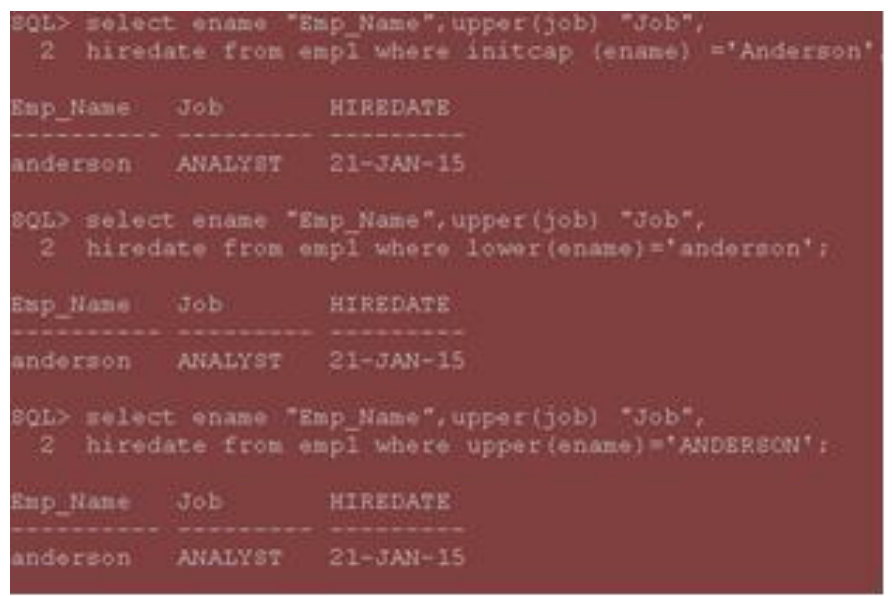

Figure 3: Where clause in Character functions

Character-Manipulation Functions:

CONCAT: Joins the strings.

SUBSTR: Extracts the measurement lengthwise of the sub string.

LENGTH: It shows how long the string is numerically.

INSTR: helps in finding the numbered position of anyalphabet which is used.

LPAD: It justified the charactersalong the right position. RPAD: It justified the characters along the left position. TRIM: Itcuts thefront and last charactersoutofa string.

TABLE 2: Character-Manipulation Functions

\begin{tabular}{|l|l|}
\hline Function & Result \\
\hline Concat('Data','Structure') & DataStructure \\
\hline Substr('Data Structure”,1,4) & Data \\
\hline Length('Data Structure”) & 13 \\
\hline Instr('DataStructure', 'S') & 5 \\
\hline Lpad(salary,5,'*') & $* * 240$ \\
\hline Rpad(salary, 5,'*') & $240^{* *}$ \\
\hline Replace('BACK and BUE','B','BL') & BLACK and BLUE \\
\hline Trim('D' from 'DataStructure') & ataStructure \\
\hline
\end{tabular}

Selectename "Name",job "Desg.", concat(ename, job) "Concate Fun." from emp1 where empno in ('1','2','3','4');

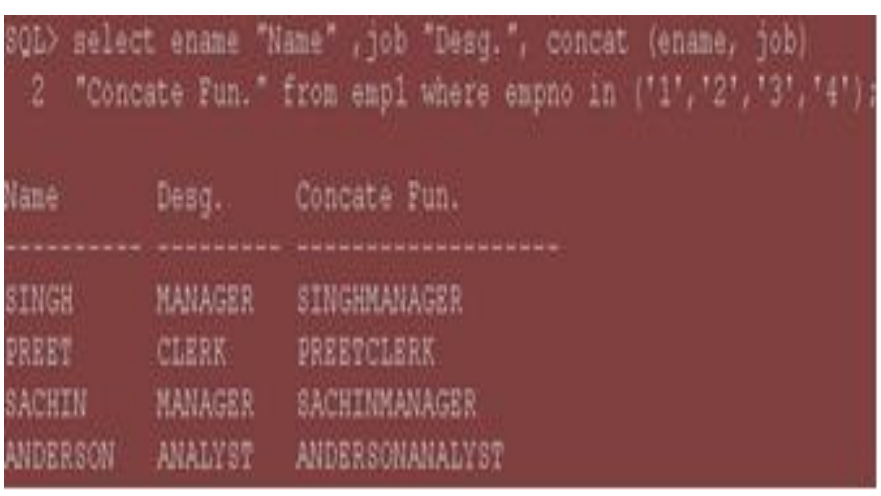

Figure 4: Concatination Function

use || symbol for cancat:

select ename "Name" ,job "Desg.", ename \|| is ' \| job

"Concate Fun." from emp1 where empno in ('1','2','3','4')

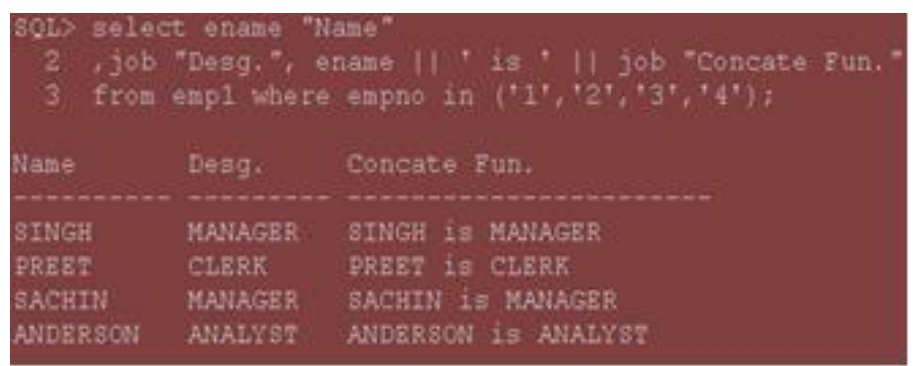

Figure 5: use || symbol for cancat

Example of Length and instr

select initcap(ename) "Name",initcap(job) "Desg.", initcap(ename) $\|^{\prime}$ is ' $\|$ initcap(job) "Concate Fun.",sal "Sal.",length(sal)

"Length_sal",instr(ename,'a')"contains'a'",instr(upper(ename) ,'A') "contains lower 'a'"from emp1 where empno in ('1','3','4','7521')

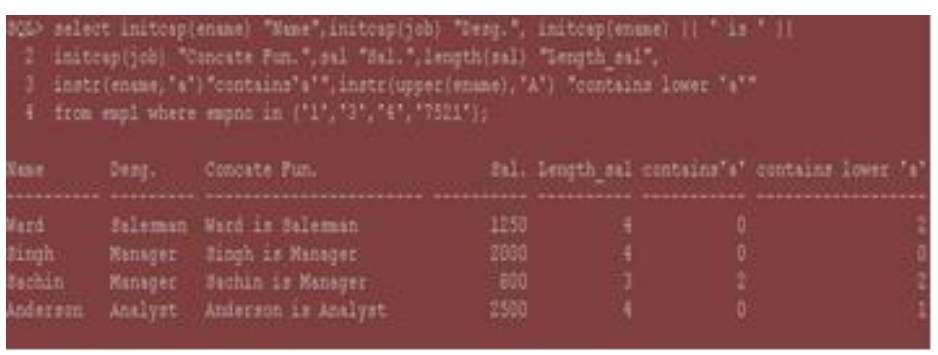

Figure 6: Example of Length and instr

Example of SUBSTR,LPAD,RPAD

Selectename,substr(ename,1,4), sal,lpad(sal,10,'\#'),rpad(sal,1 0, '\#')from emp 1 


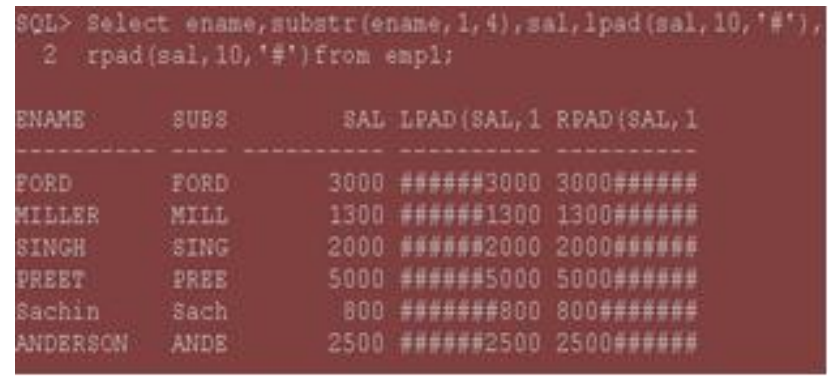

Figure 7: Example of SUBSTR,LPAD,RPAD

Example of Substr and Replace

selectename,substr(ename,1,3),replace(ename, 'a','u') from emp1 where ename like '\%a\%';

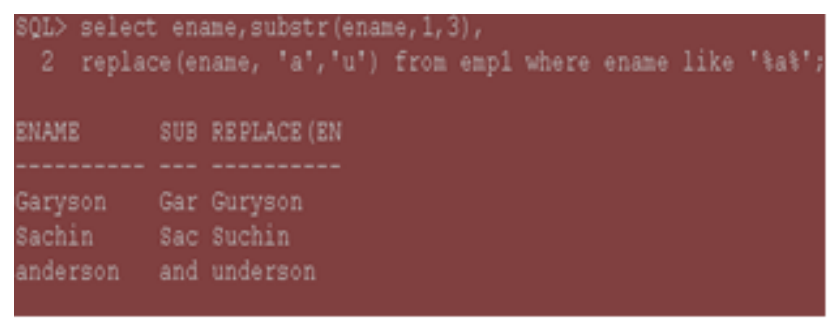

Figure 8: Example of Substr and Replace

SQL statement displays the data for those employees whose last names end with the letter $\mathrm{n}$.

Selectename, substr(ename, 1,4), length(ename),instr(ename,' $n$ ' ) from emp1 where SUBSTR(ename, -1, 1) = 'n';

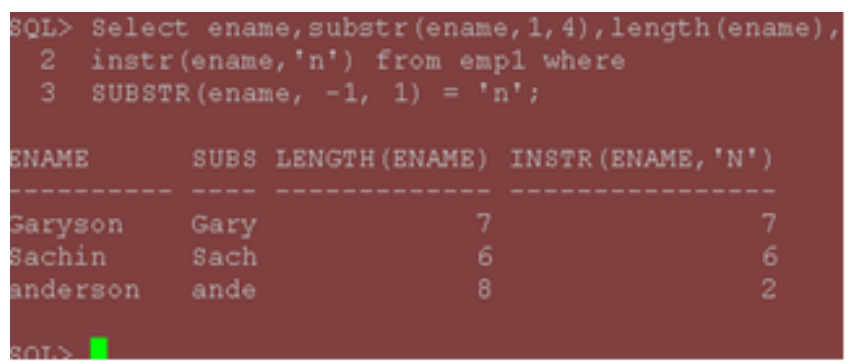

Figure 9: last names end with the letter $\mathrm{n}$.

Number Functions:

TABLE 3: Number Functions

\begin{tabular}{|c|l|}
\hline \multicolumn{1}{|c|}{ Function } & \multicolumn{1}{c|}{ Purpose } \\
\hline ROUND(column|expression, $n)$ & $\begin{array}{l}\text { This roundsoffvalues, cols and } \\
\text { numerals upto } \mathrm{n} \text { decimal places, } \\
\text { if } \mathrm{n} \text { is not included then no } \\
\text { decimal places, if } \mathrm{n} \text { is } \\
\text { unsignedthenthe numerals to left } \\
\text { position of decimal points are } \\
\text { rounded off. }\end{array}$ \\
\hline TRUNC(column|expression, $n)$ & $\begin{array}{l}\text { It eliminates the values to } \mathrm{n} \\
\text { decimal places, if } \mathrm{n} \text { is non } \\
\text { considerableit gives zero value. }\end{array}$ \\
\hline
\end{tabular}

\begin{tabular}{|l|l|}
\hline $\operatorname{MOD}(m, n)$ & Gives leftovers of $\mathrm{m}$ by $\mathrm{n}$. \\
\hline
\end{tabular}

Select $\operatorname{round}(45.923,2), \operatorname{round}(45.923,1), \operatorname{round}(45.923,-1)$, round $(44.923,-1)$ from dual;



Figure 10: Round function.

Select trunc(45.923,2), trunc(45.923,1), trunc(45.923,1),trunc(44.923,-1) from dual;

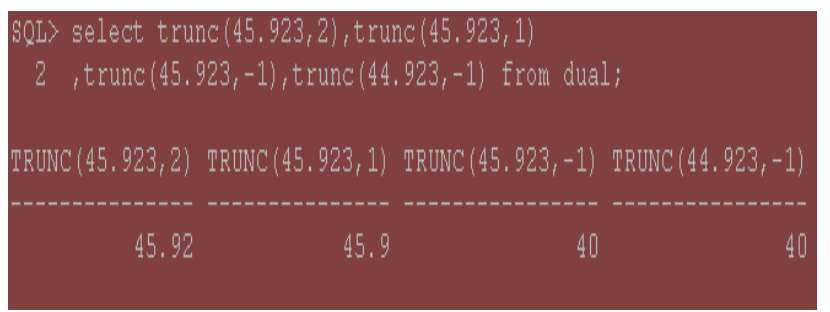

Figure 11: Trunc function

selectename "Emp_Name",sal "Sal." , MOD(sal, 1000) from emp1 where empno in ('1','2','3','4');

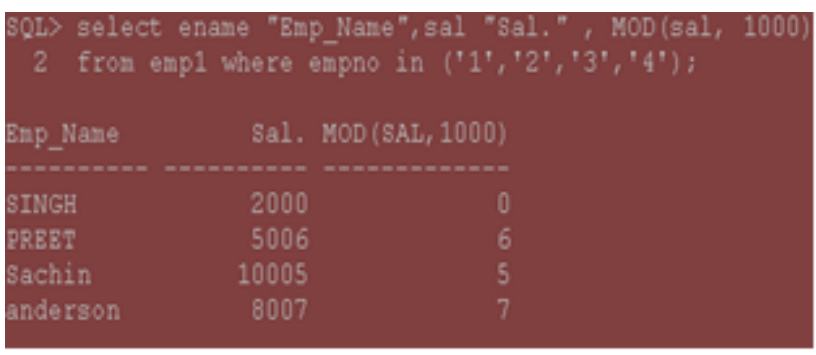

Figure 12: Mod function

Operating Dates:

The Oracle recordsdates in an interiorsyntax:

Century-year-month-day-hours-minutes- seconds.

The automatic date demonstratesyntax is DD-MON-YY selectename,hiredate from emp1; 


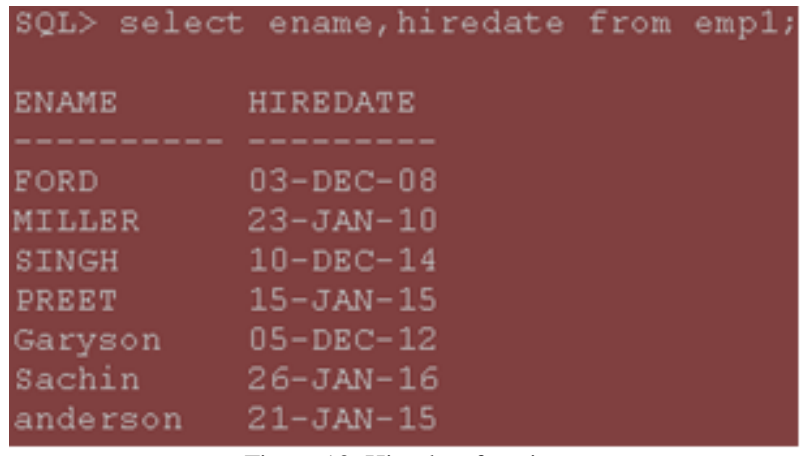

Figure 13: Hire date function

HIREDATE results as DD-MON-YY. This data is stored internally as follows:

$\begin{array}{lllllll}\text { Cen } & \text { Yr } & \text { Mon } & \text { D } & \text { Hr } & \text { Min } & \text { SEC } \\ 20 & 12 & 12 & 17 & 17 & 10 & 43\end{array}$

\section{Calculation on Dates}

1) The resultant date value can be added or subtracted to or from a date.

2) The no. of days can be calculated between two by subtracting them.

3) Hours to a date can be calculated by dividing the number of hours by 24 .

selectename "Name" ,hiredate "Hire_date",hiredate+7 "7days+hiredate" ,hiredate-7 "7days-hiredate"from emp1

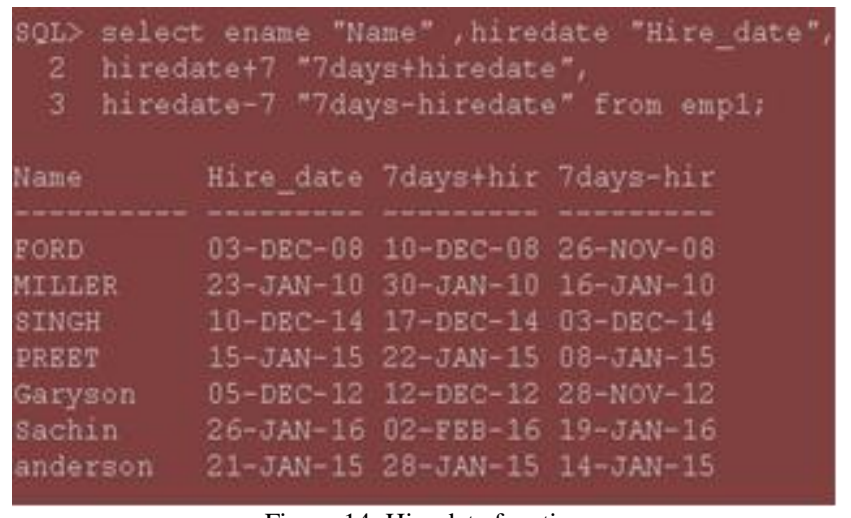

Figure 14: Hire date function

selectename "Name" ,hiredate "Hire_date",

round((sysdate-hiredate)/7,0)"No.ofweeks",round((sysdatehiredate)/30,0)"No.of months",round((sysdatehiredate)/365,0)"No.of years" from emp1.

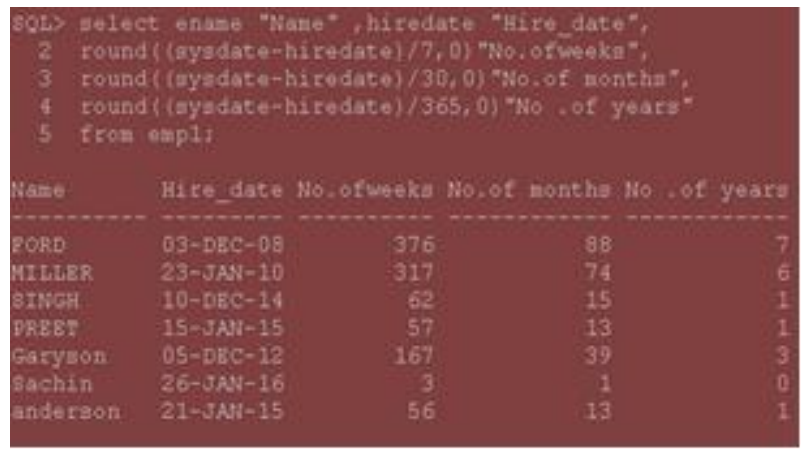

Figure 13: Round on Hire date function

Features of Date:

1)Months_Between (date1, date2):

It helps us in finding the no. of months between two dates.If date1 is afterwarddate2---output is positive; if date1 is earlier than date2, the output is negative. The non-integer portion of the output shows a segment of the month.

2)Add_Months (date, n): Add no. of months into the existing calendar date. It works only on integer values and also can be negative.

3)Next_Day(date, 'char'): Locates the next day date after the given date. It gives output in character.

4)Last_Day (date): Discovers the end date of the month while considering the given date.

5)ROUND (date [,'fmt']): Yieldsrounding of the date to specified syntax. If the syntax fmt is neglected, then date is rounded of the nearby date.

6)TRUNC (date [, 'fmt']): It yields the date after the time truncated from it. If the syntax fmt is neglected, then date is truncated to the nearby date.

Selectename,hiredate,relievingdate,round(Months_Between( relievingdate,hiredate),0)"Exp._Month",Add_Months (hiredate,,Next_Day(hiredate,'SUNDAY'),Last_Day(hiredate ) from emp1; 


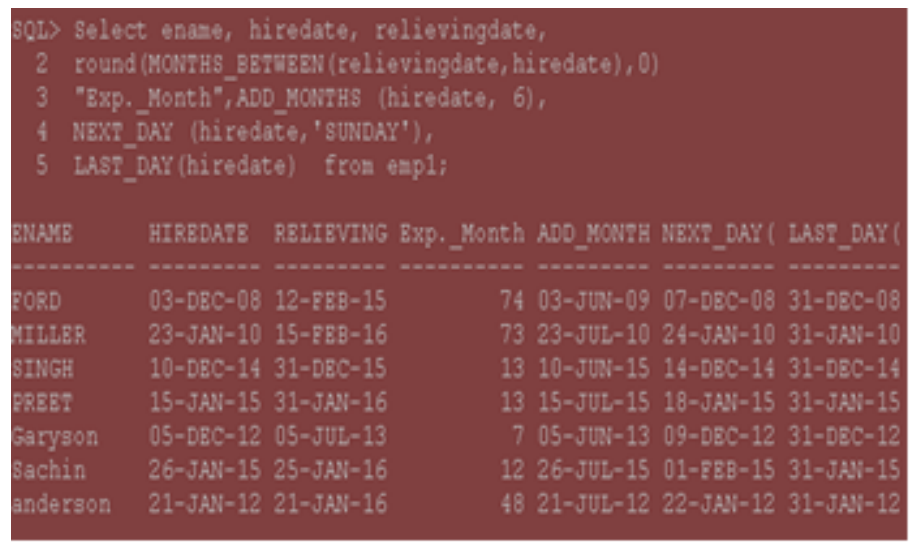

Figure 14: date function

In Where Clause

Selectename,hiredate,relievingdate,round(Months_Between (relievingdate,hiredate),0) "Exp._Month" from emp1

WhereMonths_Between (relievingdate,hiredate) $>=12$

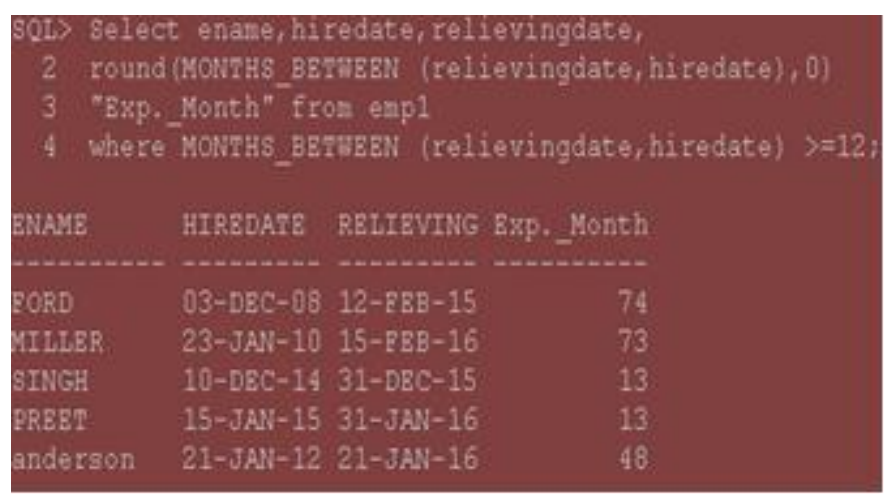

Figure 15: Hire date function with where clause

Round and Truncate Function with Dates

Select ename, hiredate, ROUND(hiredate,'MONTH'), TRUNC(hiredate,'MONTH'), ROUND(hiredate,'YEAR'), TRUNC(hiredate,'YEAR'), ROUND(hiredate,'DAY'), TRUNC(hiredate,'DAY') FROM EMP1;

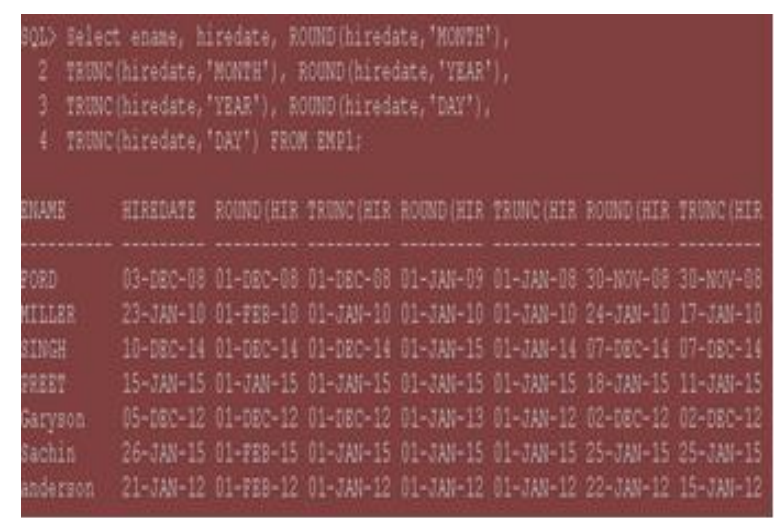

Figure 16:Round and Truncate on date

Conversion Functions
If Oracle server needs to convert one data type to the other then it can repeatedly. Converts the data to expected data type. The expected data type by the Oracle server conversion can occur wholly and clearly by the user. For this purpose some functions are required to forcefully convert the data casting to another known as conversion functions. The function names follow the conventional input data type TO output data type.

1) Conversion Type: Implicit Data Type

CHAR, VARCHAR2 can be wholly changed to NUMBER or DATE. NUMBER type value can be routinely converted to character data by Oracle server. It occurs only when the character signifies a valid number or date type value correspondingly.

For example : the select queries outputs same because Oracle inside allows 1000 and '1000' as same.

Query-1

SELECT ENAME,JOB,SAL

FROM EMP1

WHERE SAL > 15000;

Query-2

SELECT ENAME,JOB,SAL

FROM EMP1

WHERE SAL > '15000';

2) Conversion: Explicit Data Type

These functions are for single row which are skillful of converting column value, literal or an expression.

TO_DATE

TO_NUMBER

TO_CHAR

\section{3) Function: TO_CHAR}

It is required to cast a numeric input value to character type using a fixed model.

Format:

TO_CHAR(num1,[format],[nls_parameter])

Think about the below SELECT query. The query syntax the HIRE_DATE and SALARY columns of EMPLOYEES table using TO_CHAR ().

SELECT ENAME,TO_CHAR (hiredate, 'MONTH DD, YYYY') HIREDATE,TO_CHAR (sal, '\$99999.99') Salary FROM emp1 


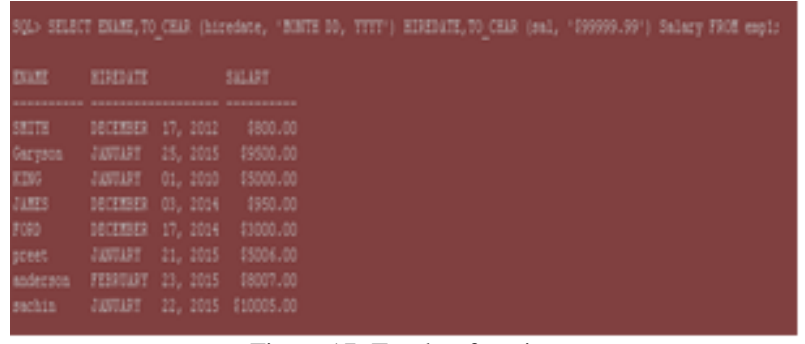

Figure 17: To-char function

TABLE 4: To-char function

\begin{tabular}{|c|c|}
\hline Syntax Model & Explanation \\
\hline ,(comma) & $\begin{array}{l}\text { This allots the position to a comma. Many no. of commas can } \\
\text { be particularlyin a number syntax model. } \\
\text { Boundaries: a number syntax model cannot begin by comma } \\
\text { element and it cannot comeat the right arrangementof a } \\
\text { decimal character or period. }\end{array}$ \\
\hline (period) & $\begin{array}{l}\text { It gives the definite position. } \\
\text { Boundaries:Itindicates only one function in a number layout } \\
\text { model }\end{array}$ \\
\hline$\$$ & Yieldsassessmentwith dollar sign. \\
\hline 0 & $\begin{array}{l}\text { It begins with zeros and proceedswith zeros at end. } \\
\text { positive-gives value with the describednumber of digits with } \\
\text { space in frontand negative with a minus sign in front. }\end{array}$ \\
\hline
\end{tabular}

\section{5) Function:TO_NUMBER}

Itconverts a numeric datatype from a character datatype.

\section{Syntax:}

TO_NUMBER(string1,[format],[nls_parameter])

list of layout models which can be used to typecast character values as number using TO_NUMBER.

\begin{tabular}{ll} 
Layout Model & \multicolumn{1}{c}{ Explanation } \\
CC & Denotes Century \\
SCC & It gives Century Before Christstarted with - \\
YYYY & It displays year havingfour numbers \\
SYYY & It gives year before Christ with-prefixed with \\
- & \\
IYYY & Gives ISO Year havingfour numbers \\
YY & It is Year having 2 digits \\
& \\
YEAR & Gives Year in alphabets \\
SYEAR & Yields Year in alphabets, BC prefixed with - \\
MONTH & Gives Month in alphabets(i.e. January) \\
MON & Results JAN, FEB \\
WW & Gives Week number (i.e. 1) \\
W & Gives Week digit of the month (i.e. 5) \\
IW & Gives Week digit of the year in ISO \\
Standard. & Results Day of years in numbers (i.e. 365) \\
DDD & Results month day in values (i.e. 28) \\
DD & Gives week day in numbers(i.e. 7) \\
D &
\end{tabular}

DAY

Monday)

FMDAY

Monday)

DY description (i.e. SUN)

Gives Day of the week in alphabets (i.e.

Gives Day of the week in characters (i.e.

HH,H12 Gives Hour number of the day (1-12)

AM, PM Gives AM or PM

MI, SS Denotes Number of minutes and seconds (i.e.

59),

SSSSS Gives seconds number of day.

DL Results Long date format. Depends on

NLS-settings. Use only with timestamp.

$\mathrm{EE} \quad$ Gives the full period name

FF Gives the fractional seconds. Use with

timestamp.

FF1..FF9 Gives the fractional seconds. Use with timestamp.

\section{FM It Fill Mode.}

FX It Format Exact: requires proper pattern matching between date and layout model.

RM Returns The Roman cipherfor month (I .. XII)

RR Returns The last 2 digits of the year.

RRRR Returns The last 2 digits of the year when used for output. Accepts fout-digit years when used for input. $\mathrm{TH}$ It Converts a integer to it's ordinal layout. For example 1 becomes 1 st.

TS Gives Short time format. Depends on NLSsettings. Use only with timestamp.

TZD It is reduced time zone name. ie PST.

TZR Denotes Time zone region

$\mathrm{X} \quad$ It Denotes Local radix character. It is a period (.) in America

The SELECT queries written beneathallow numbers as alphabet intake.

\section{SELECT TO_NUMBER('12,100.73', '999999.99') FROM DUAL;}

\section{2) Function:TO_DATE}

This acceptsalphabet values as intake and outputs theplanned date. The TO_DATE function permits users to use a date in any layout, and then it reverts the input into the default layout used by Oracle $11 \mathrm{~g}$.

Syntax:

TO_DATE( string1, [ format_mask ], [ nls_language ] ) 


\begin{tabular}{|cc|}
\hline $\begin{array}{c}\text { Layout Model } \\
\text { YEAR }\end{array}$ & Explanation \\
YYYY & It spelled outYear \\
IYY,IY,I & Gives 4-digit year \\
IYYY & Gives Last 3, 2, or 1 digit(s) of ISO year. \\
Q & It gives Quarter of year (1, 2, 3, 4; JAN-MAR = 1). \\
MM & Returns Month (01-12; JAN = 01). \\
MON & Gives name of month. \\
MONTH & Results Name of month, covering with blanksupto 9 \\
RM & characters. \\
WW & Gives Roman numerals for month starting from I-IX. \\
W & Returns Week of year (1-53) \\
IW & Gives Week of month (1-5) \\
D & On the basis of ISO standard week of year is 1-52or1-53 \\
DAY & Returns the week day. \\
DD & Gives Name of day of week. \\
DDD & Gives month day (1-31). \\
DY & Gives year day (1-366). \\
J & name of day is abbreviated \\
HH12 & Returns Julian day; \\
HH24 & Gives day hours (1-12). \\
MI,SS & Gives day hour(0-23). \\
FF & Gives Minute (0-59). \\
AM,PM & Returns seconds in fraction. \\
TZH,TZM,TZR & Gives indicator Prime Meridian \\
& \\
& Results Time zone in hour, minute. \\
\hline
\end{tabular}

Example: a character string transforms into a date syntax. SELECT TO_DATE('February 15, 1970, 11:00 A.M.', 'Month dd, YYYY, HH:MI A.M.', 'NLS_DATE_LANGUAGE $=$ American')

FROM DUAL;

\section{TO_DATE(15-FEB-70)}

Common Functions

These are used to holdvoid values in database. The purpose of the common NULL controlling function is to swap the void values with a substitute value.

\section{NVL}

The NVL -deputies another value for a void value. NVL function can be used with all kinds of data types.

\section{Syntax:}

NVL( Arg1, replace_with )

This case includesboth the constraintswhich are mandatory.

The SELECT statement will display ' $\mathrm{n} / \mathrm{a}$ ' if an employee has not been assigned any job yet i.e. JOB_ID is NULL. Else, it would exhibit the actual JOB_ID value.
SELECT first_name, NVL(JOB_ID, 'n/a')

FROM employees;

NVL2

It is an improvement over the earlier NVL,Oracle presented a facility to standby data not only for NULL columns values but also for NOT NULL columns. NVL2 can be used an alternate for Null (Void) and also for non-null value. Syntax:

NVL2( string1, value_if_NOT_null, value_if_null )

The SELECT statement under would display 'all' if the JOB_CODE for an employee is NULL. Finally, not null value of JOB CODE, it would rather display constant value 'Job done'.

SQL> SELECT NVL2(all, 'Job done', 'Bench')FROM employees;

\section{NULLIF}

The NULLIF is related to two arguments expr1 and expr2. If expr 1 equals to expr 2 then it gives NULL otherwise expr1. Dissimilar to it first parameter cannot be void.

Syntax:

NULLIF (expr1, expr2)

In this the first parameter can be nearer to NULL, but not as NULL. Both the constraints are compulsory for its execution.

The under query yields NULL until values, 16 are equal to each other.

Select NULLIF $(16,16)$ from dual;

Also, under query yields ' $\mathrm{ABC}$ ' since both the strings are not equal.

\section{SELECT NULLIF ('ABC', 'MOON')FROM DUAL;}

\section{COALESCE}

It is basic form of NVL that gives the first non-void phrase in the parameter list. It requires minimum two parameters but there is no limit on its maximum limit.

Syntax:

COALESCE (stmt1, stmt2, ...stmt_n )

Considering the SELECT query. The first not null data served into address domain for the employee.

SELECT COALESCE (address1, address2, address3) Address FROM employees;

The functioning of coalesce function is like to IF..ELSIF..ENDIF construct. 
IF address $1=!$ NULLthen

result $:=$ address 1 ;

ELSIF address $2=$ !null THEN

result $:=$ address 2 ;

ELSIF address $3=$ !null THEN

result $:=$ address 3 ;

ELSE

result := null;

END IF;

Functions: Conditional

Two functions DECODE and CASE are used in SQL statement.

1. DECODE function:

The function is similar to conditional statement IF..THEN..ELSE .

Syntax:

DECODE (exp, srch, output [, search, result $] \ldots$ [, default])

DECODE checks in sequence. If equality occurs between statement and search parameter, and it yields the conforming result. If no matches occurs then null is defined. In case types mismatch then oracle within does likely inbuilt alteration to yield the results. Oracle says two null values can be same in case of decode function.

\section{SELECT DECODE(NULL,NULL,'EQUAL','NOT EQUAL') FROM DUAL; \\ DECODE \\ EQUAL}

If NULL expression is found, then Oracle returns output of first search as null. The No. of components are 255 .

Select first_name, salary, DECODE (hire_date, sysdate,'NEW JOINEE','EMPLOYEE') FROM employees;

CASE expression

Its mechanism logically similar to DECODE but varies in format and utilization.

Syntax:

CASE [ expression ]

When 1_condition .... result_1

When 2_condition ... result_2

When $\mathrm{n}$ condition ... result $\mathrm{n}$

ELSE output

END
The determined number of parameters in a CASE expression are 255. Each WHEN ... THEN pair calculates as two arguments. To evade exceeding the limit, nested CASE expressions can be used so that the output_exp itself is a CASE expression.

Select first_name, CASE when salary $<100$ THEN 'GRADE 1'

when salary > 100 AND salary <

4000 then 'GRADE 2'

\section{ELSE 'GRADE 3'}

\section{END CASE}

From employees;

ENAM CASE

---- ------

Admin GRADE 2

Jass GRADE 3

Kumar GRADE 1

\section{CONCLUSION}

The Query processing of SQL functions comprises of conversion functions has done in this paper. This showed the data manipulation ,formatting, general functions, conditional functioning and its transformation from inbuilt to forceful conversion. In future the work can be done on multiple row functions also.

\section{REFERENCES}

[1] Groff and Weinberg, "SQL-The Complete Reference

[2] Ivan Bayross, "SQL, PL/SQL-The Programming Language of Oracle.

[3] Koch and KelvinLoney, "Oracle 9i-Complete Reference”.S

[4] Korth, "Database System Concepts".

[5] https://docs.oracle.com/cd/B19306_01/server.102/b14200/functions001. htm.

[6] https://msdn.microsoft.com/enus/library/gg415714.aspx.

[7] http://www.oracle.com/technetwork/database/bi-datawarehousing/wpsqlnaturallanguageanalysis-2431343.pdf.

[8] https://ocw.mit.edu/courses/urban-studies-and-planning/11-521-spatialdatabase-management-and-advanced-geographic-information-systemsspring-2003/lecture-notes/lect4.pdf.

[9] Jean Habimana," Query Optimization Techniques - Tips For Writing Efficient And Faster SQL Queries "International Journal of Scientific \& Technology Research volume 4, issue 10, october 2015 issn 2277-8616 22 ijstr@2015.

[10] Swati Jain and Paras NathBarwa," Performance Analysis of Optimization Techniques for SQL Multi Query Expressions Over Text Databases in RDBMS ",International Journal of Information \& Computation Technology. ISSN 0974-2239 Volume 4, Number 8 (2014), pp. 841-852.

[11] MJ Egenhofer,"Spatial SQL: A query and presentation language" IEEE Transactions on knowledge and data engineering 6 (1), 86-95.

[12] Max J Egenhofer,"Query processing in spatial-query-by-sketch" in Journal of Visual Languages \&Computing, in volume 8 and issue 4.

[13] J.Herring,R.Larsen and J.Shivakumar, "Extensions to the SQL Language to support Spatial analysis in a Topological Database" ,GIS/LIS 88,San Antonio,TX,1988,pp.741-750

[14] M.Egenhofer, “why not SQL!”,International Journal of Geographiccal Information Systems ,vo.6,no.2,pp.71-85,1992. 
[15] M.Jones, "An introduction to Gofer", Department of Computer Science,YalleUniversity,Technical report,1991.

\section{AUTHOR PROFILE}

Kamalinder Kaur is working currently as Assistant Professor in Chandigarh Engineering College, Punjab, India. She has five years of teaching experience, her research interest includes Networking with specialization in Mobile Ad-hoc Network (MANET).

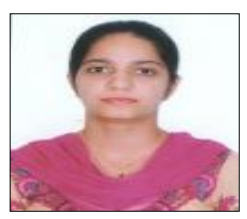

(C) 2017 by the author(s); licensee Empirical Research Press Ltd. United Kingdom. This is an open access article distributed under the terms and conditions of the Creative Commons by Attribution (CC-BY) license. (http://creativecommons.org/licenses/by/4.0/). 